\title{
EFECTOS DEL MANEJO DE SUELOS DE LADERAS EN HONGOS FORMADORES DE MICORRIZAS ARBUSCULARES Y EN BACTERIAS FIJADORAS DE NITRÓGENO EN ULTISOLES SUJETOS A EROSIÓN PLUVIAL EN LA AMAZONIA PERUANA \\ Pedro O. Ruiz ${ }^{1}$ y Charles B. Davey ${ }^{2}$
}

\begin{abstract}
Resumen
En un ensayo de vivero se evaluó la colonización con micorrizas arbusculares y la nodulación, en plantas de frejol caupí (Vigna unguiculata L. Walp) en suelos de ladera bajo cultivos en callejones (alley-cropping), cultivos continuos con bajos insumos, bosque secundario y suelo sin cobertura, a los 5, 15, 30 y 45 días después de la germinación de la semilla (DDGS). La colonización micorrícica y la nodulación presentaron una gran variación en los estados iniciales del crecimiento de las plantas de caupí a pesar de que ninguno de los sistemas estudiados recibieron fertilización fosfatada. A niveles similares y bajos de disponibilidad de fósforo en el suelo, en suelos diferentes, altos niveles de colonización micorrícica (sistema de cultivos continuos con bajos insumos) pueden ser ventajosos para el crecimiento de la planta y la nodulación. La nodulación estuvo ausente en las plantas de caupí que crecieron en el suelo de bosque secundario probablemente debido al efecto represivo de altos niveles de nitrógeno en el suelo o a la falta de rizobios para esta especie. En el suelo sin cobertura, las plantas de caupí tuvieron niveles muy bajos de colonización micorrícica. La formación de nódulos fue esencialmente nula, probablemente debido a los efectos de la pérdida de suelo por las lluvias. Los resultados obtenidos sugieren la necesidad de reintroducir los microorganismos del suelo como parte de programas de recuperación de suelos y/o revegetación de áreas severamente alteradas como los taludes de carreteras, derechos de vía de gasoductos y oleoductos, áreas expuestas por actividad minera y otras.
\end{abstract}

Palabras clave: Laderas, erosión, micorrizas arbusculares, nodulación, revegetación, Amazonía

\begin{abstract}
Arbuscular mycorrhizal colonization and nodulation in cowpea (Vigna unguiculata L.Walp) plants growing in soils under alley-cropping, low-input continuous cropping, secondary forest, and bare soil on a slope were evaluated at 5, 15, 30 and 45 days after seed germination (DASG) under greenhouse conditions. Mycorrhizal colonization and nodulation in cowpeas differed greatly at their early growth stages in spite that any of the systems studied received phosphorus fertilizers. At similar low levels of soil available phosphorus in different soils, high levels of mycorrhizal colonization (low-input continuous cropping system) may be advantageous for plant growth and nodulation. Nodulation was absent in cowpeas growing in the secondary forest soil probably due to repressing effects of high levels of nitrogen in soil or to the lack of rhizobia for cowpeas. In the bare soil plot, cowpeas had a very low level of mycorrhizal colonization. Nodule formation was essentially zero, probably as effects of soil loss due to rainfall. Results obtained suggest the need for re-introduction of soil microorganisms for revegetation and land reclamation programs in severely disturbed soils like road banks, gas and oil pipelines, mining exposed areas, and others.
\end{abstract}

Key words: Slopes, erosion, arbuscular mycorrhizae, nodulation, revegetation, Amazon

\section{Introducción}

En los trópicos húmedos bajos, la tasas de deforestación debido a la agricultura migratoria ha tenido un gran aumento en la última década debido principalmente a la gran presión demográfica y a la necesidad de producir cultivos alimenticios, afectando seriamente la productividad de los suelos por el acortamiento de los períodos de barbecho y la resultante reducción en los rendimientos de los cultivos (Sánchez et al., 1990). En la Amazonía del
Perú, esta situación se agrava por las grandes áreas dedicadas a la producción de coca (Dourojeanni, 1990).

Los suelos de la Amazonía peruana, predominantemente Ultisoles, son ácidos y deficientes en nutrientes (Sánchez, 1976) y se encuentran mayormente en áreas onduladas con laderas que tienen del 2 al $50 \%$ de pendiente (Benites, 1983). Estas áreas son particularmente propensas a una severa erosión pluvial, un problema muy serio y diseminado

\footnotetext{
${ }^{1}$ Consultor. AndeStudio S.A. Manejo de Suelos, revegetación, micorrizas. Correo electrónico: interandes@terra.com.pe

${ }^{2}$ Professor Emeritus. Soil Science and Forestry Departments. North Carolina State University. Raleigh, N.C., U.S.A.
} 
ampliamente en muchos países tropicales en desarrollo (Stocking, 1988).

Estudios conducidos en los trópicos húmedos bajos indican que la remoción del suelo superficial con la consecuente pérdida de la materia orgánica, nutrientes y la degradación de sus propiedades físicas y químicas, afectan notablemente la producción de cultivos (Alegre \& Fernández, 1991) y en muchos casos, la aplicación de fertilizantes no compensa la pérdida del suelo superficial (Lal, 1988).

El impacto de las pérdidas por erosión en las propiedades biológicas del suelo, sin embargo, no se conoce claramente. Entre estas, un importante aspecto constituye la capacidad del suelo para soportar una interacción simbiótica efectiva entre microorganismos benéficos del suelo y las raíces de las plantas. Entre las interacciones más importantes se involucra la relación simbiótica de las plantas con los hongos micorrícicos y con los rizobios (Habte, 1989; Rivera et al., 2000). Desde que el crecimiento de las plantas es afectado en forma significativa por los microorganismos del suelo, un claro entendimiento del impacto de la erosión en la productividad del suelo no se puede conseguir a menos que su impacto sobre las poblaciones microbianas sea también claramente entendido (Amaranthus \& Trappe, 1993; Franco et al. 1997; Fernandes et al., 2001; Wu et al., 2002).

En años recientes, el interés por los cultivos en callejones (alley-cropping) para la producción sostenible de alimentos y para la prevención de la pérdida del suelo en terrenos en pendientes en suelos ácidos ha aumentado en forma significativa (Palm, 1988; Szott et al., 1991a, b), donde las leguminosas constituyen un componente importante del sistema. La mayoría de las leguminosas, por otro lado, representan un caso especial entre la comunidad de plantas ya que ellas están asociadas íntimamente con micorrizas arbusculares y con rizobios.

Se conoce muy poco acerca de los efectos de la pérdida del suelo superficial en esta asociación tripartita de leguminosa-micorriza-rizobio en sistemas con bajos insumos en suelos ácidos del trópico. Investigación pertinente en Ultisoles en la Amazonía peruana no existe considerando la gran proporción de áreas agrícolas en tierra en pendientes propensas a erosión severa, especialmente cuando son manejadas de manera inapropiada.

El principal objetivo del presente estudio fue el de evaluar la colonización micorrícica y la nodulación en plantas de frejol caupí (Vigna unguiculata (L.) Walp) en Ultisoles bajo sistemas de cultivos en callejones (alley-cropping), cultivos continuos, bosque secundario y suelo sin cobertura en una pendiente en la Amazonía del Perú.

\section{Materiales y métodos}

Se condujo un experimento de vivero en la Estación Experimental de Yurimaguas, localizada en la cuenca Amazónica del Perú $\left(76^{\circ} 05^{\prime} \mathrm{O}, 05^{\circ} 45^{\prime} \mathrm{S}\right.$, a 180 m.s.n.m.). El área recibe una precipitación anual promedio de $2200 \mathrm{~mm}$ y tiene una temperatura anual promedio de $25{ }^{\circ} \mathrm{C}$. Los suelos predominantes pertenecen a la serie Yurimaguas, clasificados como franco-finos, silíceos, isohipertérmicos Paleudults Típicos.

Los suelos se colectaron del experimento de campo "Pérdidas por erosión y escorrentía en bosques, cultivos con bajos insumos y cultivos en callejones, en laderas" iniciado en el lugar mencionado en 1988. El experimento se estableció en una ladera con aspecto norte con una pendiente entre $15-20 \%$. Los tratamientos fueron instalados en parcelas de escorrentía de $22 \mathrm{~m}$ de largo por $2 \mathrm{~m}$ de ancho y fueron los siguientes: 1) cultivos continuos con bajos insumos con una rotación de arroz de secano con frejol caupí sembrados en líneas en sentido contra la pendiente (los residuos fueron dejados en la superficie del suelo); 2) líneas de guaba (Inga edulis) sembradas en sentido contra la pendiente con una separación de 4 $\mathrm{m}$. Entre las líneas se sembró una rotación de arroz de secano-frejol caupí; las podas de los árboles y los residuos se colocaron en la superficie del suelo. Se incluyeron adicionalmente dos parcelas testigo (una de bosque secundario y un suelo sin cobertura).

Se hizo una evaluación de la pérdida de suelo en el referido experimento durante el período de Diciembre de 1988 a Agosto de 1989. El área recibió $1317 \mathrm{~mm}$ de lluvia total (nueve meses) y la pérdida de suelo fue de $2.32,0.60,0.07$ y $13.00 \mathrm{Mg} \mathrm{ha}^{-1}$ para los cultivos continuos, cultivos en callejones, bosque secundario y suelo sin cobertura, respectivamente.

Para el presente experimento, se colectó suelo superficial a una profundidad de $20 \mathrm{~cm}$, de los bordes de las parcelas de escorrentía de cuatro posiciones de la ladera y luego mezclado. El suelo fue secado al aire, tamizado y mezclado. Porciones de $1.7 \mathrm{~kg}$ de suelo (peso seco) se colocaron en bolsas de plástico de $2 \mathrm{~kg}$. Las características químicas de los suelos al momento de la colecta se muestran en la Tabla 1.

El experimento tuvo un diseño de parcelas divididas con los tratamientos de manejo de suelo como parcelas principales y épocas de cosecha como sub-parcelas. Los tratamientos de manejo de suelo incluyen: 1) cultivos continuos con bajo insumos (BI); 2) cultivos en callejones (CC); 3) barbecho de bosque secundario (BS); y 4) suelo sin cobertura (SC). Las plantas se cosecharon a los 7, 15, 30 y 45 días después de la germinación de la semilla (DDGS).

Se sembraron 4 semillas de frejol caupí en cada bolsa y luego se dejó una después de la germinación. Los tratamientos de manejo de suelo tuvieron tres repeticiones por cada época de cosecha y fueron distribuidos en mesas de vivero en un diseño completo randomizado. Las plantas se regaron cuidadosamente para mantener la humedad del suelo en un $80 \%$ de su capacidad de retención de agua (por peso) y fueron re- 
distribuidas cada dos semanas para minimizar efectos posicionales.

Tabla 1. Características químicas de los suelos de los tratamientos en estudio $(0-15 \mathrm{~cm}$ de profundidad $)$.

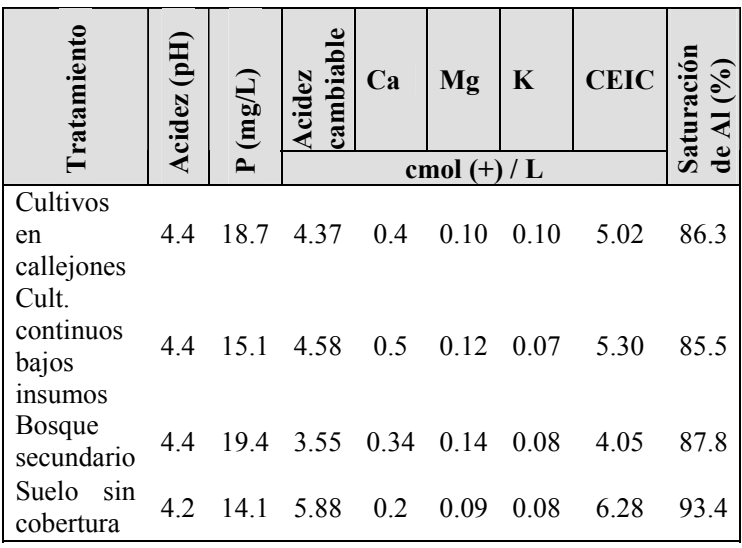

En cada época de cosecha, se evaluaron 3 plantas por tratamiento. La parte área fue cortada con tijeras a ras del suelo, secada a $70^{\circ} \mathrm{C}$ por $48 \mathrm{~h}$ y pesada. El suelo remanente y la raíz fueron colocados en bandejas de plástico donde se separó manualmente la raíz y luego se lavó. Se contaron los nódulos activos de la raíz principal. Las raíces fueron pesadas y una sub-muestra fue secada a $70^{\circ} \mathrm{C}$ durante $48 \mathrm{~h}$ para determinar el peso seco. Se colectó el $10 \%$ del peso fresco de las raíces para estimar la longitud total de la raíz y el porcentaje de colonización micorrícica, el que se determinó mediante el método de Giovanetti \& Mosse (1980) luego que las raíces fueran blanqueadas en $\mathrm{KOH}$ caliente y teñidas con azul de tripano en lacto-glicerina (Phillips \& Hayman, 1970).

Los promedios de peso seco total, y la longitud de raíces colonizadas por micorrizas, fueron comparados como tranformaciones log; los promedios del porcentaje de colonización micorrícica como tranformaciones arcsin; los promedios de nodulación como transformaciones de raíz cuadrada $x+1$. Los datos transformados fueron luego analizados mediante el procedimiento ANOVA del Sistema de Análisis Estadístico (SAS) (1988). A menos que se indique lo contrario, se usó un nivel de significación de $\mathrm{p}<0.01$ para comparar los promedios debido a una variabilidad anticipada de los suelos. El efecto lineal del fósforo en la biomasa total, nodulación, porcentaje de colonización micorrícica y longitud de raíces con micorriza, se probaron usando el procedimiento GLM (SAS, 1988).

\section{Resultados y discusión}

La diferencia en el porcentaje de colonización micorrícica entre los tratamientos de manejo de suelo fue significativa a través del tiempo $(p=0.0027)$. El porcentaje de colonización micorrícica en el sistema de bajos insumos (BI) fue significativamente mayor que en el de cultivos en callejones (CC), bosque secundario (BS) y suelo sin cobertura (SC) $(\mathrm{p}=$ 0.0002), respectivamente (Figura 3). La longitud total de raíces con micorrizas fue también significativamente más grande que en el trt. BI que en los otros trts. $(\mathrm{p}=0.002)$, , pero la interacción de este parámetro con la época de cosecha no fue significativa (Figura 2). Los niveles más altos de colonización micorrícica encontrados en el tratamiento BI, con relación a los otros tratamientos, se deben probablemente a la presencia de especies más efectivas o compatibles de hongos MA en el suelo. En el campo, es probable que durante aproximadamente 4 años de cultivos continuos de caupí en rotación con arroz de secano, haya ocurrido una selección natural de hongos micorrícicos adecuados para el caupí (Abbott \& Robson, 1991)

En el tratamiento $\mathrm{CC}$, en condiciones de campo, el caupí también fue incluido como un cultivo rotacional, sin embargo, es posible que haya ocurrido una asociación preferencial hongo MA / planta hospedera a favor de Inga edulis la que se sembró en líneas como parte del sistema. Esta asociación preferencial es posible que ocurra probablemente debido a la mayor permanencia del árbol en el campo. En el trt. BS, los niveles bajos de colonización encontrados a lo largo del experimento, están probablemente relacionados a la falta de un simbionte adecuado para el caupí, teniendo en cuenta que la mayoría de especies en los barbechos de bosque secundario en la región de Yurimaguas están altamente colonizados por micorrizas.

Los niveles muy bajos de colonización micorrícica en el trt. SC es posible que estén relacionados a otros factores diferentes a los del mismo bosque. Estos factores se relacionan a los efectos de la pérdida de suelo por su exposición permanente, sin una cobertura vegetal, a la ocurrencia de lluvias durante aproximadamente 4 años (Carpenter et al., 2001). La pérdida de suelo fue de $13 \mathrm{Mg} \mathrm{ha}^{-1}$ en un período de 9 meses (Diciembre de 1988 a Agosto de 1989) (Alegre \& Fernández, 1991). En los trópicos húmedos bajos, los propágulos de los hongos MA se encuentran mayormente en la capa hojarasca (Rose \& Paranka, 1987; Rivera, 2003) y en el suelo superficial $(20 \mathrm{~cm})$ donde proliferan las raíces absorbentes (Redhead, 1977). Entonces es probable que las poblaciones de hongos se hayan reducido como consecuencia de la pérdida de suelo. Las plantas de caupí en este suelo, sin embargo, tuvieron colonización micorrícica leve, debido probablemente a la presencia de raíces colonizadas de otras plantas en áreas adyacentes o a la ocurrencia de propágulos de micorrizas a profundidades mayores por debajo del suelo superficial (Zajicek et al., 1986).

El análisis de variancia de la biomasa total no mostró diferencias significativas para todos los tratamientos con el tiempo. El peso total de las plantas de caupí sin embargo, fue más alto en el trt. BI en los 
otros tratamientos a los 45 DDGS (Figura 2). El crecimiento de las plantas en los tratamientos BS y SC fueron los más bajos entre los tratamientos estudiados.

Se encontraron diferencias significativas en la nodulación entre los tratamientos $(\mathrm{p}=0.0007)$ y también con el tiempo $(\mathrm{p}=0.0001)$. No se observó nodulación en ninguno de los tratamientos No se observó nodulación en ninguno de los tratamientos a los 7 y 15 DDGS (Figura 3). A los 30 DDGS se observaron nódulos solamente en los tratamientos de cultivos en callejones y sistema de bajos insumos (1 y 4 , respectivamente) y similarmente a los 45 DDGS (2 y 7 , respectivamente). Se debe señalar que en general, la nodulación en estos tratamientos fue más baja de lo que se esperaba. La presencia de rizobios compatibles en estos dos tratamientos probablemente dieron cuenta por la nodulación observada. Esto es posible debido a que el caupí fue sembrado durante 4 años antes que los suelos se colectaran para el presente experimento.

La nodulación estuvo ausente por completo en los tratamientos de bosque secundario y suelo sin cobertura. En el tratamiento de bosque secundario la ausencia de nódulos se debió probablemente a la falta de rizobios adecuados para el caupí en el suelo, resultando en la inhibición de la actividad de los rizobios por los altos contenidos de nitrógeno en el suelo, de acuerdo con Vitousek \& Sanford (1986) y Palm \& Meléndez (1991). En el caso el suelo sin cobertura, la ausencia de nódulos, se debe posiblemente a los efectos de la erosión al reducir las poblaciones de rizobios o a la baja disponibilidad de nutrientes, principalmente fósforo y calcio $\mathrm{y}$ a la acidez y contenidos altos de aluminio (Aziz \& Habte, 1990).

El efecto lineal del fósforo no fue significativo para la biomasa total de la planta, el porcentaje de colonización micorrícica y la longitud de raíces con micorrizas. Más bien fue significativo solamente para la nodulación $(\mathrm{p}=0.0812)$. Esta prueba puede indicar que a niveles similares de $\mathrm{P}$ en el suelo, la producción más alta de biomasa en trt. BI con relación a los otros tratamientos, se debió a los efectos de los niveles más altos de colonización micorrícica. Es posible también que los altos niveles de colonización en el trt. BI influyó en la nodulación del caupí.

Se debe señalar que los valores de producción de biomasa y de nodulación en las plantas de caupí en el trt. BI presente experimento fueron comparables con los obtenidos en un sistema de bajos insumos de un experimento previo en fechas de cosecha similares. Asimismo, a los 45 DDGS, no se observó floración en ninguno de los tratamientos, tal como se observó en un tratamiento con fertilización. Estas observaciones sugieren que los niveles de $\mathrm{P}$ en el suelo probablemente no son los más adecuados para el crecimiento del caupí.

\section{Conclusiones}

El presente bioensayo mostró los efectos del manejo del suelo en una ladera en el crecimiento, colonización micorrícica y nodulación en caupí. A pesar de que ninguno de los tratamientos estudiados recibió aplicaciones de fertilizantes, los niveles de colonización micorrícica y de nodulación tuvieron grandes diferencias en los estados iniciales del crecimiento del caupí. A niveles similares de $\mathrm{P}$ disponible en el suelo en suelos diferentes, niveles altos de colonización micorrícica pueden ser ventajosos para el crecimiento de la planta y para la nodulación. La formación de nódulos en el bosque secundario estuvo ausente debido probablemente a los altos niveles de nitrógeno en este suelo o a la falta del rizobio adecuado para el caupí. La pérdida de suelo en las áreas en laderas por efectos de la erosión pluvial parece afectar no sólo las propiedades físicas y químicas del suelo sino también las biológicas, tales como las poblaciones de hongos micorrícicos y bacteria rizobiales. La conducción de este tipo de bioensayos puede ser útil para evaluar la ocurrencia de la colonización micorrícica y la nodulación en especies leguminosas seleccionadas para sistemas con bajos insumos en suelos ácidos y deficientes en $\mathrm{P}$ en los trópicos húmedos bajos. Asimismo, los resultados obtenidos, indican la necesidad de introducir las poblaciones microbianas al suelo en programas de recuperación de tierras y/o revegetación de áreas severamente alteradas tales como los taludes de las carreteras, derechos de vía de oleoductos y gasoductos, áreas expuestas por la actividad minera y otras (Rosales et al., 1997; Raja et al., 2001; Dodd et al., 2002; Cuenca et al., 2002).

\section{Agradecimientos}

El presente estudio constituye un capítulo de la Tesis de Grado para optar el Doctorado en Ciencias del Suelo en la Universidad Estatal de Carolina del Norte. El autor agradece al Dr. Charles B. Davey, a la Misión Carolina del Norte y al personal profesional de la Estación Experimental de Yurimaguas, Loreto.

\section{Literatura citada}

Abbott L.K. \& Robson A.D. 1991. Factors influencing the occurrence of vesicular-arbuscular mycorrhizas. Agric. Ecosyst. and Environ. 35: 121-150.

Alegre J.C. \& Fernandes E.C.M. 1991. Runoff and erosion losses under forest, low-input and alleycropping in slopes. pp. 227-228. In: T. McBride (Ed.). TropSoils Technical Report 1988-1989. North Carolina State University. Raleigh, North Carolina.

Amaranthus M.P. \& Trappe J.M. 1993. Effects of erosion on ecto- and VA-mycorrhizal inoculum potential of soil following forest fire in southwest Oregon. Pl. Soil. 150: 41-49.

Aziz T. \& Habte M. 1990. Stimulation of mycorrhizal activity in Vigna unguiculata through low level 
fertilization of an Oxisol subjected to imposed erosion. Commun. in Soil Sci. Planta Anal. 21: 493505.

Benites J.R. 1983. Alternativas para los terrenos abandonados del cultivo de la coca en el Alto Huallaga. CIPA XVI-Estación Experimental de Yurimaguas. Programa de Suelos Tropicales. Serie de Separatas $\mathrm{N}^{\circ} 4$. Yurimaguas, Perú.

Carpenter F.L., Mayorga S.P., Quintero E.G. \& Schroeder M. 2001. Land use and erosion of a Costa Rican Ultisol affect soil chemistry, mycorrhizal fungi and early regeneration. Forest Ecology and Management. 144(1-3): 1-17.

Cuenca G., De Andrade Z., Lovera M., Meneses E., Márquez M. \& Machuca R. 2002. The use of mycorrhizal native shrubs in the rehabilitation of degraded areas from La Gran Sabana State. Venezuela. Interciencia. 27(4): 165.

Dodd J.C., Dougall T.A., Clapp J.C. \& Jeffries P. 2002. The role of arbuscular mycorrhizal fungi in plant community establishment at Samphire Hoe, Kent, UK - the reclamation plattform created during the building of the Channel tunnel between France and UK. Biodiversity and Conservation. 11(1): 39.58.

Dourojeanni M.J. 1990. Amazonía: Qué hacer? Centro de Estudios Teológicos de la Amazonía (CETA). Iquitos, Perú.

Fernández E.C.M., Styger E. \& Rakotondramasy H. 2001. Mycorrhiza fungal diversity as impacted by the loss of endemic, rainforest plants in Madagascar. ICOM3. Adelaide, Australia.

Franco A.A. \& DeFaria S.M. 1997. The contribution of $\mathrm{N}-2$ fixing tree legumes to land reclamation and sustainability in the tropics. Soil Biol. Biochem. 29(5-6): 897-903.

Giovanetti M. \& Mosse B. 1980. An evaluation of techniques for measuring vesicular-arbuscular mycorrhizal infection on roots. New Phytol. 84: 489500 .

Habte M. 1989. Impact of simulated erosion on the abundance and activity of indigenous vesiculararbuscular mcorrhizal endophytes in an Oxisol. Biol. Fert. Soils. 7: 164-167.

Lal R. 1988. Soil erosion research on steep lands. In: pp. 45-53. W.C. Moldenhauer and N.W. Hudson (Eds.). Conservation Farming on Steep Lands. SWCS, Ankeny, IA.

Palm C.A. 1988. Mulch quality and nitrogen dynamics in an alley cropping system in the Peruvian Amazon. $\mathrm{Ph}$. D. Dissertation. Soil Science Dept. North Carolina State University. Raleigh, NC. U.S.A.

Palm C.A. \& Meléndez G. 1991. Sustainable agriculture: soil carbon and nitrogen dynamics. In: T. McBride (Ed.). TropSoils Annual Report 19881989. North Carolina State University. Raleigh, North Carolina. : 205-206.

Phillips J.M. \& Hayman D.S. 1970. Improved procedures for clearing roots and staining parasitic and vesicular-arbuscular mycorrhizal fungi for rapid assessment of infection. Trans. Brit. Mycol. Soc. 55:158-161.

Raja P., Herdhawati J., Nazam C.A. \& Zubaidah I. 2001. Rehabilitation of Malasyan highway landscape plants using commercial arbuscular mycorrhizal fungi. ICOM3. Adelaide, Australia.

Redhead J.F. 1977. Endotrophic mycorrhizas in Nigeria: species of the Endogonaceae and their distribution. Trans. Brit. Mycol. Soc. 69: 275-280.

Rivera E.L. 2003. Presencia de estructuras características de hongos micorrícicos arbusculares en hojas en procesos de descomposición ¿Se puede considerar un hecho común en la naturaleza?. Unidad de Biotecnología Vegetal. Universidad Javeriana. Bogotá, Colombia.

Rivera E.L., Campos R. \& De Martínez A.L. 2000. Mycotrophic status of wax laurel useful for the recuperation of eroded soils in Tomine Reservoir in Colombia. Suelos Ecuatoriales. 30 (2): 188-193.

Rosales J.G., Cuenca G., Ramírez N. \& Andrade Z. 1997. Native colonizing species and degrades land restoration in La Gran Sabana, Venezuela. Restoration Ecology. 5(2): 147.155.

Rose S.L. \& Paranka J.E. 1987. The location of roots and mycorrhizae in tropical forest litter. $\underline{\text { In: D.M. }}$ Sylvia, L.L. Hung, and H.J. Graham (Eds.). Mycorrhizas in the Next Decade. Proc. 7th North American Conference on Mycorrhizae (NACOM). Gainesville, Florida.

Sanchez P.A. 1976. Properties and management of soils in the tropics. Wiley, New York.

Sanchez P.A., Palm C.A. \& Smyth T.J. 1990. Approaches to mitigate tropical deforestation by sustainable soil management practices. Developments in Soil Science. 20: 211-220.

Statistical Analysis System. 1988. SAS/STAT User's Guide. SAS Institute, Cary, NC, USA.

Stocking M.A. 1988. Socio-economics of soil conservation in developing countries. J. Soil Water Conserv. 43: 381-385.

Szott L.T., Palm C.A. \& Sanchez P.A. 1991a. Agroforestry in acid soils in the humid tropics. Adv. Agron. 45: 275-301.

Szott L.T., Fernandes E.C.M. \& Sanchez P.A. 1991b. Soil-plant interactions in agroforestry systems. For. Ecol. Mgmt. 45: 127-152.

Vitousek P.M. \& Sanford R.L. 1986. Nutrient cycling in moist tropical forest. Ann. Rev. Ecol. 17: 136167.

Zajicek J.M., Daniels Hetrick B.A. \& Owensby C.E. 1986. The influence of soil depth on mycorrhizae colonization of forbs in the tallgrass prairie. Mycologia. 76: 316-320.

Wu T.H., Hao W.Y., Lin X.G. \& Shi Y.Q. 2002. Screening of arbuscular mycorrhizal fungi for the revegetation of eroded red soils in subtropical China. Plant and Soil. 239 (2): 25-235. 


\section{Anexos}
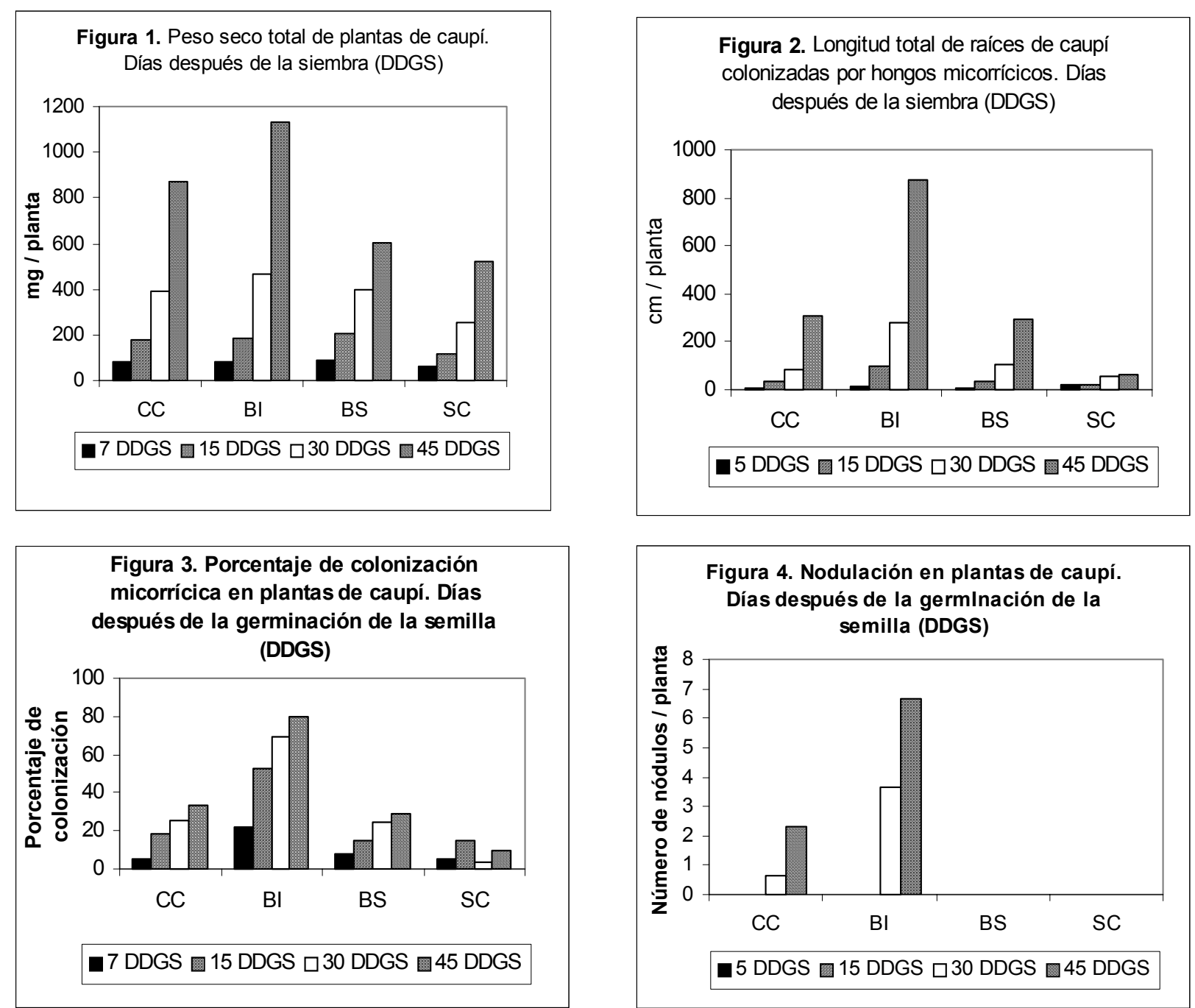

SE1 = Error estándar para una diferencia de promedios entre dos fechas para un tratamiento (raíz cuadrada 2MSEb / 3)

SE2 = Error estándar para comparar dos de estas diferencias entre tratamientos (raíz cuadrada 4MSEb / 3)

Figura 1. $\mathrm{SE} 1=0.18 \quad \mathrm{SE} 2=0.25$

Figura 2. $\mathrm{SE} 1=0.12 \quad \mathrm{SE} 2=0.17$

Figura 3. $\mathrm{SE} 1=4.51 \quad \mathrm{SE} 2=6.38$

Figura 4. $\mathrm{SE} 1=0.08 \quad \mathrm{SE} 2=0.11$ 